\title{
A fatal case of primary melioidotic prostatic abscess: the peril of poor drug compliance
}

\author{
Wahab, A.A. ${ }^{1 *}$, Norliyana, N. ${ }^{1,2}$, Ding, C.H. ${ }^{1}$, Nurzam, S.C.H. ${ }^{2}$, Salbiah, N. ${ }^{2}$ and Rao, K.R. ${ }^{3}$ \\ ${ }^{1}$ Department of Medical Microbiology and Immunology, Faculty of Medicine, Universiti Kebangsaan Malaysia, \\ 56000 Cheras, Kuala Lumpur, Malaysia \\ ${ }^{2}$ Department of Pathology, Kuala Lumpur General Hospital, 50586 Kuala Lumpur, Malaysia \\ ${ }^{3}$ Department of Urology, Selayang Hospital, 68100 Batu Caves, Selangor, Malaysia \\ *Corresponding author e-mail: saw@ppukm.ukm.edu.my \\ Received 6 November 2019; received in revised form 4 May 2020; accepted 6 May 2020
}

\begin{abstract}
Primary prostatic melioidosis is a rare presentation of melioidosis even in melioidosis endemic areas. We report a case of a 58-year-old man with underlying diabetes mellitus who presented with a 5-day history of high-grade fever associated with lower urinary tract symptoms. Suprapubic tenderness and tender prostatomegaly were noted on examination. An abdominal computed tomography (CT) scan confirmed the presence of a prostatic abscess. Both blood and prostatic pus cultures grew Burkholderia pseudomallei. He was initially started on intravenous ceftazidime, followed by an escalation to intravenous meropenem. He was discharged home with oral amoxicillin-clavulanate and doxycycline after completing 12 days of meropenem. Unfortunately, his compliance to oral antibiotic therapy was poor, and he succumbed to the disease.
\end{abstract}

INTRODUCTION

Melioidosis is caused by a saprophytic gramnegative bacillus known as Burkholderia pseudomallei. This bacterium is commonly found in moist soil and pooled surface water. Northeast Thailand, Malaysia, Singapore, and northern Australia are considered highly endemic areas (Dance, 2000). The pathogenesis of this infection is mainly based on its ability to invade phagocytes and survive intracellularly (Wiersinga et al., 2012). Lungs are the main organ involved in this infection but extrapulmonary manifestations do occur occasionally (How et al., 2005). Involvement of the prostate is rare, particularly as a primary focus of melioidosis. Prostatic abscesses have been reported to occur in about $13 \%$ of the cases, as primary and secondary foci of melioidosis (Kingsley et al., 2016).

\section{CASE REPORT}

A 58-year-old Malay man with underlying type 2 diabetes mellitus presented with highgrade fever for 5 days. This was associated with suprapubic pain and dysuria. He did not complain of any other symptoms. He was a retired factory supervisor. Since retiring, he spent his time gardening around his house. On clinical examination, he was febrile with a temperature of $38.5^{\circ} \mathrm{C}$ and tachycardic with a pulse rate of 130 beats/ min. His blood pressure and glucometer readings were $130 / 90 \mathrm{mmHg}$ and 6.4 umol/L, respectively. He had tenderness at the suprapubic region. A digital rectal examination revealed an enlarged and tender prostate. The rest of the clinical examination was unremarkable.

Laboratory investigations revealed the presence of leukocytosis, with a predominance of neutrophils (87.9\%), a high 
C-reactive protein and pyuria. Other laboratory results were within their reference ranges, as shown in Table 1 . His chest X-ray was unremarkable, with no radiological evidence of a pulmonary infection. A transrectal ultrasound showed the presence of hypoechoic lesions in the prostate suggestive of an abscess, as shown in Figure 1. CT scans of the pelvis and abdomen were performed to confirm the lesions. The CT scan revealed an enlarged prostate with a localized hypodense lesion measuring 55 mm x $54 \mathrm{~mm}$ x $69 \mathrm{~mm}$, which was consistent with an abscess. There was no radiological evidence of a hepatic or splenic abscess.

A decision was made to drain the prostatic abscess and a transrectal ultrasound (TRUS)-guided needle aspiration was performed. The pus specimen was sent for bacterial culture. Both pus and blood samples (taken earlier) grew B. pseudomallei that was susceptible to ceftazidime, meropenem, trimethoprim-sulfamethoxazole (TMP-SMX), amoxicillin-clavulanic acid and tetracycline, as shown in Table 2. The identification of Burkholderia pseudomallei was accomplished biochemically using the API $20 \mathrm{NE}$ kit (bioMérieux, France). A urine specimen was also sent for culture but it yielded no growth.

He was empirically started on IV ceftazidime $2 \mathrm{~g}$ q8h, which was continued after the culture results were available on Day-3 of admission. However, he did not demonstrate significant clinical improvement after five days of therapy. His condition deteriorated further with the development of respiratory distress, necessitating intensive care unit admission. However, repeated chest X-rays did not show any new or significant changes. His sputum was also sent for microbiological culture, but no bacterial growth was recorded. Repeated blood cultures were still positive for $B$. pseudomallei, with an antibiogram similar to that of the initial isolate. The antimicrobial therapy was escalated to IV meropenem $1 \mathrm{~g}$ q8h. His fever gradually subsided and he was subsequently weaned off oxygen supplementation. He completed 12 days of meropenem therapy and was discharged home with oral amoxicillin-clavulanate $1 \mathrm{~g}$ bd and oral doxycycline $200 \mathrm{mg}$ od for three months. Oral TMP-SMX was not prescribed

Table 1. Laboratory investigation results on the day of presentation

\begin{tabular}{lcc}
\hline Parameters & Result & Normal Range \\
\hline Full Blood Count: & & \\
$\quad$ Hemoglobin & 12.3 & $11.6-15.1 \mathrm{~g} / \mathrm{dL}$ \\
White cell count & $12.9 \times 10^{9} / \mathrm{L}$ & $4.1-11.4 \times 10^{9} / \mathrm{L}$ \\
Platelet & $215 \times 10^{9} / \mathrm{L}$ & $171-399 \times 10^{9} / \mathrm{L}$ \\
\hline C-reactive protein & 11.8 & $<0.5 \mathrm{mg} / \mathrm{dL}$ \\
\hline Liver Function Test: & & \\
Total Protein & 68 & $64-83 \mathrm{~g} / \mathrm{L}$ \\
Albumin & 41 & $35-50 \mathrm{~g} / \mathrm{L}$ \\
Alkaline Phosphatase & 62 & $0-55 \mathrm{U} / \mathrm{L}$ \\
$\quad$ Alanine transaminase & 21 & \\
\hline Renal profile: & & $136-145 \mathrm{mmol} / \mathrm{L}$ \\
Sodium & 138 & $3.5-5.2 \mathrm{mmol} / \mathrm{L}$ \\
Potassium & 4.5 & $3.2-7.4 \mathrm{mmol} / \mathrm{L}$ \\
Urea & 5.2 & \\
Creatinine & 77.9 & \\
\hline Urinalysis: & & \\
Red blood cell & Negative & \\
Leukocyte & Positive (2+) & \\
Nitrate & Negative & \\
Glucose & Negative & \\
Ketone & Negative & \\
\hline
\end{tabular}




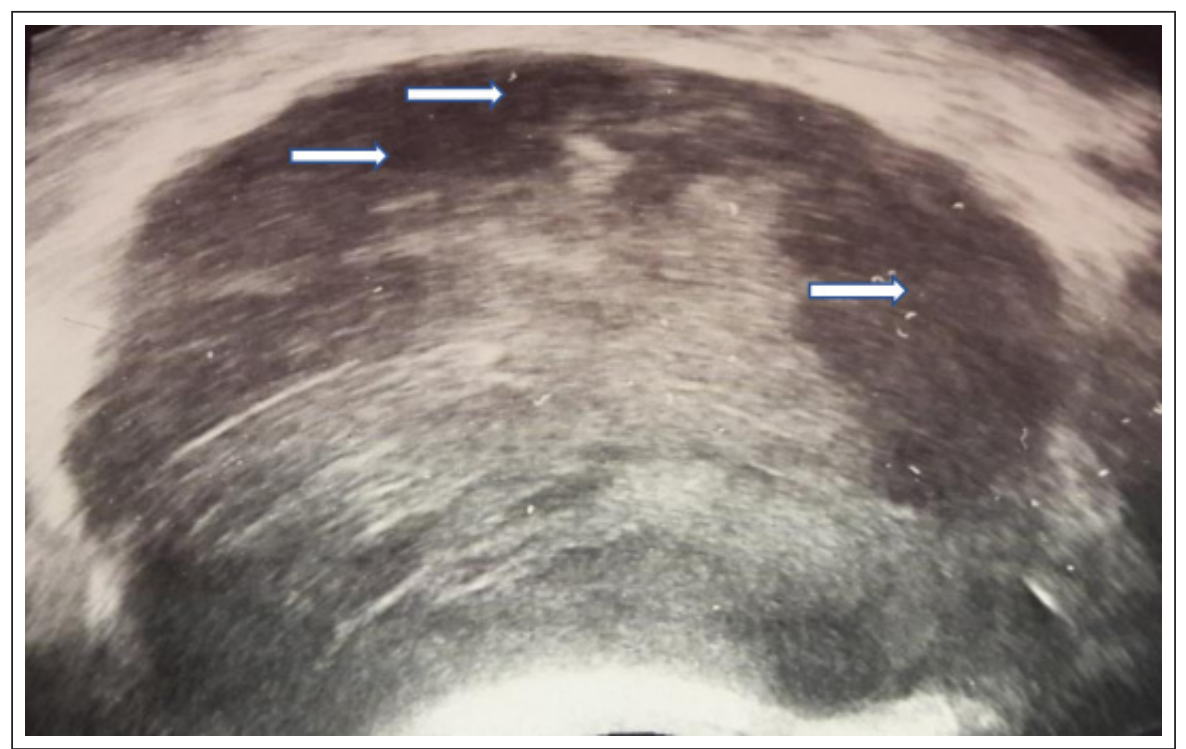

Figure 1. Ultrasound of the prostate showing the presence of an abscess (arrows).

Table 2. Antibiotic susceptibility testing result for this Burkholderia pseudomallei isolate

\begin{tabular}{lcccc}
\hline \multirow{2}{*}{ Antibiotic } & $\begin{array}{c}\text { Minimum inhibitory } \\
\text { concentration (MIC) } \mu \mathrm{g} / \mathrm{mL}\end{array}$ & \multicolumn{2}{c}{ MIC Range Reference $(\mu \mathrm{g} / \mathrm{mL})^{*}$} \\
\cline { 3 - 5 } & 2 & 8 & 16 & 32 \\
Amoxicillin-clavulanic acid & 1.5 & 8 & 16 & 32 \\
Ceftazidime & 0.38 & 4 & 8 & 16 \\
Imipenem & 2 & 2 & - & 4 \\
Trimethoprim-sulfamethoxazole & 2 & 4 & - & - \\
(TMP-SMX) & 2 & & Resistant \\
Doxycycline & 2 & & \\
\hline
\end{tabular}

* Based on the Clinical and Laboratory Standards Institute (CLSI) Methods for Antimicrobial Dilution and Disk Susceptibility Testing of Infrequently Isolated or Fastidious Bacteria. $3^{\text {rd }}$ ed. CLSI guideline M45.

because the patient was allegedly allergic to this antibiotic.

Three months after being discharged home, the patient did not turn up for his scheduled follow up in Kuala Lumpur General Hospital. It was learnt that the patient was not compliant to his antibiotics and was admitted to another hospital. Alas, he succumbed to the infection.

\section{DISCUSSION}

The incidence of melioidosis varies widely between regions, depending on its geographical and agriculture activities. Its incidence in Malaysia was reported to be between 6.1 to 16.35 cases per 100,000 population in the various states (How et al., 2005; Hassan et al., 2010). The infection is acquired mainly through inhalation or direct penetration of a pre-existing skin condition. Thus, occupational and recreational activities that have direct or regular contact with contaminated soil or water are considered risk-related activities (Hassan et al., 2010; Currie et al., 2010). Diabetes mellitus is one of the most important risk factors associated with melioidosis, as previously reported by many authors (How et al., 2005; Zueter et al., 2016). Coming back to our patient, his underlying diabetes 
mellitus and regular soil exposure through gardening activities were his most likely risk factors for contracting melioidosis.

Melioidosis may mimic many other conditions as multi-organ involvement is common. An acute presentation is reported in the majority of cases, with bacteraemia being a common occurrence (Kingsley et al., 2016; Currie et al., 2010). Similarly, our patient had acute symptoms and bacteraemia. Although genitourinary involvement in melioidosis is not common, prostatic abscess has been reported before (Kingsley et al., 2016; Currie et al., 2010). A prostatic abscess in melioidosis frequently presents as a secondary infection (as part of systemic melioidosis) rather than a primary focus. Hassan et al. (2010) and Zueter et al. (2016) described only one case of primary melioidotic prostatic abscess in their series. Additionally, Kingsley et al. (2016) demonstrated only three cases of primary prostatic melioidosis from 56 cases. Thus, prostatic abscess as the primary focus of melioidosis is exceedingly rare.

Most prostatic abscess patients present with urinary tract symptoms that include dysuria, urgency, frequency, retention or incontinence (Kozlowska et al., 2018; Tan et al., 2015). Kozlowska et al. (2018) concluded that the absence of urinary symptoms had a negative predictive value of $96 \%$ for prostatic abscess. However, Chong et al. (2014) showed that only two out of their nine patients had dysuria. A digital rectal examination can be inconclusive, because a tender prostate is elicited in less than half of prostatic abscess patients (Kozlowska et al., 2018). The presence of leukocyturia was perhaps the most consistent finding - all melioidosis patients with prostatic abscess had urinary leukocyte counts of at least $50 \times 10^{6} / \mathrm{L}$, and the value of $<50 \times 10^{6} / \mathrm{L}$ correlated with a $100 \%$ negative predictive value (Kozlowska et al., 2018). Echoing these findings, our patient also had a classical history of urinary symptoms, a tender enlarged prostate and significant leukocyturia.
TRUS and CT scan modalities are useful imaging investigations. In most cases, they will reveal large abscesses $(>4.5 \mathrm{~cm}$ ) (as was seen in our patient), involvement of both lobes and multiloculated abscesses (Chong et al., 2014; Tan et al., 2002). Microbiological culture will help to confirm B. pseudomallei. A pus specimen drained from the abscess is perhaps the most useful specimen for bacterial culture (Tan et al., 2015; Tan et al., 2002; Chee \& Chee, 2018). The bacterium can also be isolated from urine specimens and blood specimens in some patients (Tan et al., 2015; Tan et al., 2002). In our patient, the bacteria was isolated from both pus and blood specimens, but not from the urine.

The role of surgery for this condition remains controversial. It is suggested that a small abscess of less than $1 \mathrm{~cm}$ may be managed conservatively with antimicrobial therapy (Acherman et al., 2018) whereas a larger abscess should be promptly drained (Tan et al., 2002). Antimicrobial treatment of melioidosis can be divided into an initial intensive phase of at least two weeks on an intravenous antibiotic, followed by an eradication phase of at least 3 months on oral antibiotics (Pitman et al., 2015). Ceftazidime is considered the first-line intravenous antibiotic but the switch to carbapenem can be contemplated in the event of ceftazidime failure (Limmathurotsakul $\&$ Peacock, 2011). The suggested maximum dosages for ceftazidime and meropenem are $2 \mathrm{~g}$ and $1 \mathrm{~g}$, respectively, every 8 hours for a minimum duration of 10-14 days (Limmathurotsakul \& Peacock, 2011). Coming back to our patient, he was given the maximum ceftazidime dosage but clinical improvement was not apparent and in fact was complicated by respiratory failure secondary to sepsis. Thus, the switch to a carbapenem was made, and he was successfully treated with meropenem for 12 days.

It was noted that a relapse or recurrence can occur in melioidosis. Cultureconfirmed recurrences were reported in $11.4 \%$ of patients who survived their initial 
hospitalisation in Singapore (Chien et al., 2018). A recurrence is more likely to occur in those with multifocal disease and/or an intraabdominal abscess (Chien et al., 2018). The choice of oral therapy has been shown to influence relapse rates. A study has shown that the use of amoxicillin-clavulanate as well as TMP-SMZ alone and doxycycline alone was associated with higher relapse rates (Limmathurotsakul et al., 2006). In the same study, it was observed that the duration of oral therapy was negatively associated with relapse. The hazard ratio decreased by $29 \%$ for each 4 -week increase in the duration of standard oral treatment (Limmathurotsakul et al., 2006). Thus, it is important to give emphasis on the choice and duration of oral therapy during the maintenance phase. Our patient was appropriately prescribed with a combination of oral amoxicillin-clavulanate and doxycycline (as he was allergic to TMP-SMZ). Alas, although both the drug dosages and treatment duration were adequate, his drug compliance was poor, leading to a possible relapse and ultimately his demise.

\section{CONCLUSION}

The diagnosis of primary melioidotic prostatic abscess remains a diagnostic and therapeutic challenge. The diagnosis should be considered in diabetic patients with urinary tract symptoms. Pharmacological treatment consists of two phases, with the total treatment duration taking several months in order to prevent a relapse. Drug compliance is particularly paramount during the second (i.e. maintenance) phase.

\section{Conflict of interest}

The authors declare that they have no conflict of interest.

Acknowledgement. The authors express their sincere gratitude to the Director General of Health, Ministry of Health Malaysia and Dean, Faculty of Medicine, Universiti Kebangsaan Malaysia for their permission to publish this case report.

\section{REFERENCES}

Ackerman, A.L., Parameshwar, P.S. \& Anger, J.T. (2018). Diagnosis and treatment of patients with prostatic abscess in the post-antibiotic era. International Journal of Urology 25(2): 103-110.

Chee, Y.C. \& Chee, Y.N. (2018). An unusual case of primary melioidotic prostatic abscess complicated by perianal abscess. ID Cases 11: 51-52.

Chien, J.M., Saffari, S.E., Tan, A.L. \& Tan, T.T. (2018). Factors affecting clinical outcomes in the management of melioidosis in Singapore: a 16-year case series. $B M C$ Infectious Diseases 18: 482.

Chong, V.H., Sharif, F. \& Bickle, I. (2014). Urogenital melioidosis: a review of clinical presentations, characteristic and outcomes. Medical Journal of Malaysia 69(6): 257-60.

Currie, B.J., Ward, L. \& Cheng, A.C. (2010). The epidemiology and clinical spectrum of melioidosis: 540 cases from the 20 year Darwin prospective Study. PLOS Neglected Tropical Diseases 4(11): e900.

Dance, D.A. (2000). Melioidosis as an emerging global problem. Acta Tropica 74(2-3): 115-9.

Hassan, M.R., Pani, S.P., Peng, N.P., Voralu, K., Vijayalakshmi, N., Mehanderkar, R., Aziz, N.A. \& Michael, E. (2010). Incidence, risk factors and clinical epidemiology of melioidosis: a complex socio-ecological emerging infectious disease in the Alor Setar region of Kedah, Malaysia. BMC Infectious Diseases 10: 302.

How, S.H., Ng, T.H., Jamalludin, A.R., Tee, H.P., Kuan, Y.C., Alex, F., Aminudin, C.A., Sapari, S. \& Quazi, M.H. (2009). Pahang melioidosis registry. Medical Jourmal of Malaysia 64(1): 27-30.

Kingsley, P.V., Leader, M., Nagodawithana, N.S., Tipre, M. \& Sathiakumar, N. (2016). Melioidosis in Malaysia: A review of case reports. PLOS Neglected Tropical Diseases 10(12): e0005182. 
Kozlowska, J., Smith, S., Roberts, J., Pridgeon, S. \& Hanson, J. (2018). Prostatic abscess due to Burkholderia pseudomallei: Facilitating diagnosis to optimize management. The American Journal of Tropical Medicine and Hygiene 98(1): 227-230.

Limmathurotsakul, D., Chaowagul, W., Chierakul, W., Stepniewska, K., Maharjan, B., Wuthiekanun, V., White, N.J., Day, N.P. \& Peacock, S.J. (2006). Risk factors for recurrent melioidosis in northeast Thailand. Clinical Infectious Diseases 43(8): 979-86.

Limmathurotsakul, D. \& Peacock, S.J. (2011). Melioidosis: a clinical overview. British Medical Bulletin 99: 125-139.

Pitman, M.C., Luck, T., Marshall, C.S., Anstey, N.M., Ward, L. \& Currie, B.J. (2015). Intravenous therapy duration and outcomes in melioidosis: a new treatment paradigm. PLOS Neglected Tropical Diseases 9(3): e0003586.
Tan, G., Li, J., Chen, L., Chen, X., Zhang, S., Ke, L. \& Liu, J. (2015). Prostatic melioidosis rarely reported in China: two cases report and literatures review. International Journal of Clinical and Experimental Medicine 8(11): 21830-2.

Tan, J.K., Yip, S.K., Png, D.J. \& Moorthy, P. (2002). Primary melioidotic prostatic abscess: presentation, diagnosis and management. ANZ Journal of Surgery 72(6): 408-10.

Wiersinga, W.J., Currie, B.J. \& Peacock, S.J. (2012). Melioidosis. The New England Journal of Medicine 367(11): 1035-44.

Zueter, A., Yean, C.Y., Abumarzouq, M., Rahman, Z.A., Deris, Z.Z. \& Harun A. (2016). The epidemiology and clinical spectrum of melioidosis in a teaching hospital in a North-Eastern state of Malaysia: a fifteen-year review. $B M C$ Infectious Diseases 16: 333. 